\title{
Force Field Based Joint Optimization of Strictly Monotonic KPIs in Wireless Networks
}

\author{
Sascha Berger, Albrecht Fehske, and Gerhard Fettweis \\ Vodafone Stiftungslehrstuhl \\ Technische Universität Dresden, Germany \\ Email: $\{$ sascha.berger, albrecht.fehske, fettweis $\} @$ ifn.et.tu-dresden.de
}

\begin{abstract}
Self-organizing networks (SONs) are expected to improve the quality of service $(\mathrm{QoS})$ while reducing the operator's capital and operational expenditures. In order to achieve this ambitious goal, SON concepts are required to manage multiple key performance indicators (KPIs) by modifying several different network control parameters. Developing concepts and algorithms that cope with such complexity is not easy. In order to avoid the complexity of joint optimization, the research so far focused on concepts, which coordinate multiple algorithms [1], [2]. Usually, each of these algorithms is dedicated to managing a single use case or KPI. In contrast, we introduce and analyse a force field based joint optimization concept which considers all KPIs and network control parameters concurrently. Despite the holistic approach, our concept offers an acceptable complexity. Using an algorithm based on this concept, we can resolve a high load scenario by jointly adjusting the tilts and cell individual offsets (CIOs).
\end{abstract}

Index Terms-Self-Organizing Networks, Joint Optimization, Coordinated Optimization, Force Field, Load Balancing.

\section{INTRODUCTION}

Network operators expect SONs to reliably reduce their operational and capital expenses while improving the network's QoS. Aiming for this ambitious goal, SONs need to consider an extensive variety of KPIs while adjusting various network control parameters (short: parameters). As a result, a major challenge of future SONs is their enormous complexity. Thus, it is not surprising that current approaches try to simplify the problem by dividing the task into multiple use cases [1], [2]. Employing a separate algorithm for each use case, comes at the added cost of necessitating a coordination of these algorithms. Moreover, obtaining optimal trade-offs is very challenging. Each algorithm may optimize one or a small subset of all KPIs while neglecting other KPIs. If a neglected KPI behaves contrary to the KPIs considered for a particular parameter, the QoS can be worsened by the usage of the algorithm. In order to drive all KPIs into their desired value ranges, various algorithms are employed successively what requires an additional coordination algorithm. Let us consider a simple example: We take an algorithm dedicated to optimizing the coverage. Such an algorithm may increase the transmit power of a site in order to cover the maximum area possible. However, increasing the transmit power may also increase interference with neighbouring sites. Hence, the algorithm optimizes coverage while worsening the interference situation. Subsequently, an algorithm which is designed to optimize the interference needs to be employed until both coverage and interference are within their desired value ranges. In recent work, researchers explore joint optimization approaches as they expect these approaches to outperform coordination concepts while dealing with higher complexity [3]. In contrast to coordination concepts, joint optimization concepts consider all relevant KPIs simultaneously, i.e. one algorithm optimizes all relevant KPIs. In this paper we

- Propose a force field based joint optimization concept for SONs (section II-B). The concept uses ideas and laws from classical physics in order to modify $m$ parameters concurrently while considering $n$ KPIs. Despite using such a holistic approach this concept remains at an easily manageable complexity level. Moreover, this concept contains the ability to seamlessly balance trade-offs as desired by the operator.

- Investigate the convergence behaviour of the concept proposed in II-C. Using Zangwill's convergence theorem, we can state that an algorithm based on the concept proposed will converge to a point in the solution set if a certain convergence condition is fulfilled.

- Analyse the solution set. We show that under the given conditions, all possible solutions are Pareto optimal (II-D). Furthermore, we discuss the results obtained in II-E.

- Employ the proposed concept to balance a high load scenario (section III) by simultaneously adjusting the cell individual offsets (CIOs) and the antenna tilts. Apart from adjusting the load, we will also consider the 5 th percentile of the signal-to-interference-and-noise-ratio (SINR) and obtain fundamental trade-offs between the latter and an optimal load balancing.

We conclude our work in section IV.

\section{ForCE FIELD BASED APPROACH FOR SON}

\section{A. Initial Situation}

Let us consider the downlink of a cellular network with $L$ cells. At each cell, the SON-algorithm modifies $M$ parameters in order to manage $N$ KPIs. The parameters and KPIs considered are the same at every cell. We use the following definitions:

- All $N \cdot L$ KPI values are successively written into a vector $\boldsymbol{K} \in \mathcal{Y} \subset \mathbb{R}^{N L}$, where the coordinates $K_{1} \ldots K_{N}$ contain 
the KPI values of the first cell, $K_{N+1} \ldots K_{2 N}$ the KPI values of the second cell and so forth. All $K_{n}$ are scaled to cover the range from 0 to 100 .

- All parameter values are successively written into a vector $\boldsymbol{P} \in \mathcal{X} \subset \mathbb{R}^{M L}$, where the coordinates $P_{1} \ldots P_{M}$ contain the parameter values of the first cell, $P_{M+1} \ldots P_{2 M}$ the parameter values of the second cell and so forth. All $P_{m}$ are also scaled from 0 to 100 .

- The mapping $\Omega: \mathcal{X} \subset \mathbb{R}^{M L} \rightarrow \mathcal{Y} \subset \mathbb{R}^{N L}$ maps the system setting (the $M \cdot L$ parameters) to the KPIs: $\boldsymbol{\Omega}(\boldsymbol{P})=\boldsymbol{K}$. In general $\boldsymbol{\Omega}$ is unknown. However, $\boldsymbol{\Omega}$ always fulfills: $\boldsymbol{K}^{\prime}=\boldsymbol{K}$ if $\boldsymbol{P}^{\prime}=\boldsymbol{P}$. $\boldsymbol{\Omega}_{n}$ maps the system setting to $K_{n}: \boldsymbol{\Omega}_{n}(\boldsymbol{P})=K_{n}$. We assume that $\boldsymbol{\Omega}$ and $\boldsymbol{\Omega}_{n}$ are differentiable with respect to $\boldsymbol{P}$.

- $\boldsymbol{\Phi}(\boldsymbol{K}) \in \mathbb{R}^{M L \times N}$ is the potential matrix:

$$
\boldsymbol{\Phi}=\left(\begin{array}{ccc}
\Phi_{1,1}\left(K_{1}\right) & \ldots & \Phi_{1, N}\left(K_{N}\right) \\
\vdots & \ddots & \vdots \\
\Phi_{M L, 1}\left(K_{1}\right) & \ldots & \Phi_{M L, N}\left(K_{N}\right)
\end{array}\right)
$$

Since we consider the same $N$ KPI-types at each cell, we refer to the $N$ KPIs at cell $l$ also as $K_{1} \ldots K_{N}$ instead of $K_{(l-1) N+1} \ldots K_{l N}$. Note that we guarantee uniqueness already by means of the indices of $\Phi_{m, n}$. According to $\boldsymbol{\Phi}(\boldsymbol{K})$, we have for each of the $M \cdot L$ parameters $N$ potentials. Thus, each potential is with respect to a specific parameter at a certain cell (rows) and a certain KPI (columns). Moreover, we require $\Phi_{m, n}$ to be convex and differentiable with respect to $K_{n}$.

\section{B. Concept}

The central idea of our concept is to create situationdependent forces which act on the network's parameters leading to a force-dependent parameter modification. As in physics, each force $F_{m, n}$ is induced by an associated potential $\Phi_{m, n}\left(K_{n}\right)$ as follows

$$
F_{m, n}:=-\gamma_{m, n} \cdot \nabla_{K_{n}} \Phi_{m, n}\left(K_{n}\right) .
$$

In the formula above, $\nabla_{K_{n}} \Phi_{m, n}\left(K_{n}\right)$ is the gradient of $\Phi_{m, n}$ with respect to $K_{n} . \gamma_{m, n}$ is a non-negative scaling factor. The potentials express the operator's goals, i.e. they are flat (independent of $K_{n}$ ) at desired KPI values and show a gradient if $K_{n}$ is not within the desired range. Since we also require convexity for the potentials, it is given that a force is negative if the KPI is higher than desired and positive if the KPI is smaller than desired. The potentials may be interpreted as cost functions as well. Each force $F_{m, n}$ acts at its corresponding parameter $m$. Hence, $N$ different forces try to change the same parameter in order to drive their KPI into the desired value range. According to Eq. (2), a higher gradient leads to a stronger force which results in a larger parameter change. The overall parameter change that arises is defined as

$$
\Delta \boldsymbol{P}:=\boldsymbol{F}:=\left(\begin{array}{c}
-\sum_{n=1}^{N} \gamma_{1, n} \nabla_{K_{n}} \Phi_{1, n} \\
\vdots \\
-\sum_{n=1}^{N} \gamma_{M L, n} \nabla_{K_{n}} \Phi_{M L, n}
\end{array}\right) .
$$

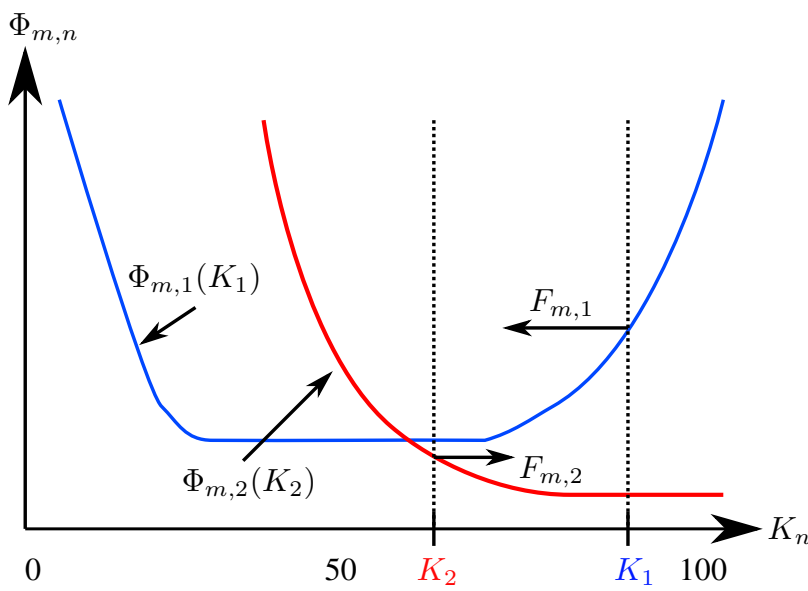

Fig. 1. Two exemplary potentials for a certain cell and a certain parameter.

All forces which act on the same parameter interfere with each other, leading to the overall force vector $\boldsymbol{F} \in \mathbb{R}^{M L}$. Changes in the parameters result in KPI changes as well. The concept proposed operates iteratively, i.e. in the next iteration step the forces will be updated and act on the parameters yet again. Let us examine an example for a specific cell, which has a single variable parameter $m$. The operator wants to balance two different KPIs. In Fig. 1, exemplary potentials are presented. We can directly obtain the optimization goals from the potentials, e.g. KPI 2 should have a larger value than approx. 80. If we now imagine that the KPIs have the values as indicated by the dotted lines in Fig. 1, we would have two counteracting forces. Each force tries to change the parameter $m$ to its own advantage. However, according to the potentials it holds that, $\left|F_{m, 1}\right|>\left|F_{m, 2}\right|$. Following this, we obtain that the acting force $F_{m}=\sum_{n=1}^{2} F_{m, n}$ is in the direction of $F_{m, 1}$, given that $\gamma_{m, 1}=\gamma_{m, 2}$. Nevertheless, the induced parameter change is weakened due to a counteracting effect of KPI 2.

The system reaches a stable state if $\boldsymbol{F}=0$. This is the case if either all single forces $F_{m, n}$ are zero, i.e. if all KPIs are within their desired value range, or if several counteracting forces cancel each other out. The latter case means that a trade-off is present. A SON-algorithm based on this concept may be dedicated to running permanently, i.e. it runs forever. We analyse this approach and give a detailed discussion in the following sections. The idea to employ forces for a joint optimization is inspired by [4], where a force field approach is used for optimal base station (BS) placement.

\section{Convergence Analysis}

In order to evaluate an algorithm it is important to know which kind of solutions we obtain by using the algorithm and under which conditions we obtain these results. In order to analyse the latter property for an algorithm based on the concept proposed, we examine a convergence analysis following Zangwill's Global Convergence Theorem [5] in this section. We will analyse the solution set in section II-D. Prior to the convergence analysis, we will introduce an algorithm following the force field approach explained above. 
Let $\mathcal{A}\left(\boldsymbol{K}^{j}\right)=\boldsymbol{K}^{j+1}$ be the iterative algorithm as defined below. The algorithm $\mathcal{A}$ is expected to run permanently and to balance

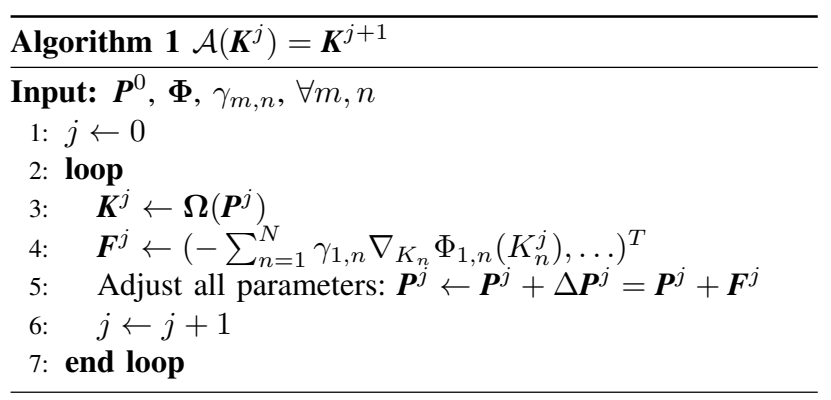

all KPIs considered. Let us define the solution set $\Gamma$ as follows

$$
\Gamma:=\{\boldsymbol{K} \mid \boldsymbol{F}=0\} .
$$

In the following convergence analysis, we want to investigate the conditions under which the sequence $\{\boldsymbol{K}\}_{j=0}^{\infty}$, generated by $\mathcal{A}$, converges to an element in the solution set. According to [5], the limit of any convergent subsequence of $\{\boldsymbol{K}\}_{j=0}^{\infty}$ is a solution if

1) the sequence $\{\boldsymbol{K}\}_{j=0}^{\infty} \subset \mathcal{S}$ and $\mathcal{S}$ is a compact set,

2) the mapping $\mathcal{A}\left(\boldsymbol{K}^{j}\right)=\boldsymbol{K}^{j+1}$ is closed, and

3) there is a continuous function $\mathcal{Z}$ such that $\mathcal{Z}\left(\boldsymbol{K}^{j+1}\right)<$ $\mathcal{Z}\left(\boldsymbol{K}^{j}\right)$ for $\boldsymbol{K}^{j} \notin \Gamma$ and $\mathcal{Z}\left(\boldsymbol{K}^{j+1}\right) \leq \mathcal{Z}\left(\boldsymbol{K}^{j}\right)$ for $\boldsymbol{K}^{j} \in \Gamma$.

Condition 1 For $\mathcal{S}:=\left\{\boldsymbol{K}: 0 \leq K_{n} \leq 100, \forall n\right\}$ the sequence $\{\boldsymbol{K}\}_{j=0}^{\infty}$ is a subset of $S$. By definition, $\mathcal{S}$ is bounded and closed. Hence, it is a compact set.

Condition 2 The algorithm $\mathcal{A}$ maps $\mathcal{S}$ to $\mathcal{S}$ and can be written as $\mathcal{A}\left(\boldsymbol{K}^{j}\right)=\boldsymbol{K}^{j+1}=\boldsymbol{\Omega}\left(\boldsymbol{P}^{j+1}\right)=\boldsymbol{\Omega}\left(\boldsymbol{P}^{j}+\boldsymbol{F}^{j}\right)$. In section II-A, we already assumed that $\boldsymbol{\Omega}$ is differentiable, thus, $\boldsymbol{\Omega}$ is continuous. As $\mathcal{S} \subset \mathbb{R}^{N L}$ is an euclidean space, it is a Hausdorff space as well. Hence, $\mathcal{A}$ must be closed [6].

Condition 3 We will chose the overall weighted potential

$$
\varphi=\sum_{n=1}^{N} \sum_{m=1}^{M L} \gamma_{m, n} \Phi_{m, n}\left(K_{n}\right)
$$

as the continuous function $\mathcal{Z}$. If $\boldsymbol{K}^{j} \in \Gamma$, it follows that $\boldsymbol{F}^{j}=\Delta \boldsymbol{P}^{j}=0$. Since we already assumed that $\boldsymbol{K}^{j+1}=\boldsymbol{K}^{j}$ if $\boldsymbol{P}^{j+1}=\boldsymbol{P}^{j}$, we immediately obtain $\varphi\left(\boldsymbol{K}^{j+1}\right)=\varphi\left(\boldsymbol{K}^{j}\right)$ for $\boldsymbol{K}^{j} \in \Gamma$. In case $\boldsymbol{K}^{j} \notin \Gamma$, condition 3 is satisfied if $\left\langle\nabla_{\boldsymbol{P}} \varphi, \Delta \boldsymbol{P}\right\rangle<0$ and the step size chosen is sufficiently small [7]. The latter can be directly influenced by the non-negative scaling factors $\gamma_{m, n}$. We can define them as $\gamma_{m, n}:=\kappa \cdot \hat{\gamma}_{m, n}$, where $\hat{\gamma}_{m, n}$ accounts for the scaling among the forces and the global step length $\kappa$ always ensures a small enough step length. Note that in algorithm $\mathcal{A}$ the step length $\kappa$ is an input variable (via $\gamma_{m, n}$ ) independent of the iteration $j$. However, we could also set the step length $\kappa$ in every iteration. $\nabla_{\boldsymbol{P}} \varphi$ can be written as

$$
\nabla_{\boldsymbol{P}} \varphi=\sum_{n=1}^{N} \sum_{m=1}^{M L} \gamma_{m, n} \underbrace{\nabla_{\boldsymbol{P}} \Phi_{m, n}\left(K_{n}(\boldsymbol{P})\right)}_{=*} .
$$

In the formula above, $*=\left(\frac{\partial \Phi_{m, n}}{\partial K_{n}} \frac{\partial K_{n}}{\partial P_{1}}, \ldots, \frac{\partial \Phi_{m, n}}{\partial K_{n}} \frac{\partial K_{n}}{\partial P_{M L}}\right)^{T}$. $K_{n}$ is given by the mapping $\boldsymbol{\Omega}_{n}(\boldsymbol{P})$. Thus, we can write $\frac{\partial K_{n}}{\partial P_{r}}=\frac{\partial \boldsymbol{\Omega}_{n}}{\partial P_{r}}$. Following this, we can now obtain a general convergence condition for the algorithm $\mathcal{A}$ as

$$
\left\langle\nabla_{\boldsymbol{P}} \varphi, \boldsymbol{F}\right\rangle=\sum_{r=1}^{M L} \sum_{n=1}^{N} \sum_{m=1}^{M L} \gamma_{m, n} \frac{\partial \Phi_{m, n}}{\partial K_{n}} \frac{\partial \boldsymbol{\Omega}_{n}}{\partial P_{r}} \cdot F_{r}<0 .
$$

Since Eq. (7) is rather involved, let us write the convergence condition for the simple example introduced above $(M=1, N=2, L=1)$ :

$$
\left[\gamma_{1} \frac{\partial \Phi_{1}}{\partial K_{1}} \frac{\partial \boldsymbol{\Omega}_{1}}{\partial P}+\gamma_{2} \frac{\partial \Phi_{2}}{\partial K_{2}} \frac{\partial \boldsymbol{\Omega}_{2}}{\partial P}\right] \cdot F<0
$$

We know that $F \cdot \gamma_{1} \frac{\partial \Phi_{1}}{\partial K_{1}}<0$ and $F \cdot \gamma_{2} \frac{\partial \Phi_{2}}{\partial K_{2}}>0$. Furthermore, we know that $\left|\gamma_{1} \frac{\partial \Phi_{1}}{\partial K_{1}}\right|>\left|\gamma_{2} \frac{\partial \Phi_{2}}{\partial K_{2}}\right|$. Hence, in this example $\mathcal{A}$ converges for sure, if $\frac{\partial \boldsymbol{\Omega}_{1}}{\partial P}=\frac{\partial \boldsymbol{\Omega}_{2}}{\partial P}>0$. Now, it is obvious that in general $\mathcal{A}$ converges, if

$$
\frac{\partial \boldsymbol{\Omega}_{n}}{\partial P_{r}}=\frac{\partial \boldsymbol{\Omega}_{u}}{\partial P_{v}}>0 \forall n, r, u, v .
$$

This implies that all KPIs $K$ are required to 1) increase if a parameter increases, and 2) to increase with the same slope. Since the inequality in Eq. (9) is coherent with the mathematical definition of a strictly monotonic increasing function, we referred to it in the title as strictly monotonic KPI. An example for a strictly monotonic KPI could be the reference-signal-receive-power-coverage as a function of the transmit power. Condition 2) seems to be rather strong. Nevertheless, it should be pointed out that the convergence condition in Eq. (9) is much stronger than required. In general, Eq. (7) provides the weakest convergence condition.

\section{Solution Set Analysis}

Now that we know the conditions under which the algorithm $\mathcal{A}$ converges to an element in the solution set $\Gamma$ (defined in Eq. (4)), let us analyse the solution set itself. $\Gamma$ includes situations where 1) all $F_{m, n}$ are zero, and 2) where all components of $\boldsymbol{F}$ are zero. Case 1) implies that all KPIs are within their desired value range. Thus, 1) equals the optimal solution. In case 2), several forces which act on the same parameter $m$, cancel each other out, i.e. at least two KPIs are not within their desired value range. Hence, we denote solutions according to case 2) as trade-off solutions. But what kind of trade-offs are these solutions? Before answering this, let us first introduce the concept of a Pareto optimal solution [8].

Definition A solution $\hat{\boldsymbol{K}}$ is called efficient or Pareto optimal, if there is no other $\boldsymbol{K}$ such that $\Phi_{m, n}\left(K_{n}\right) \leq \Phi_{m, n}\left(\hat{K}_{n}\right) \forall m, n$ and $\Phi_{m, n}\left(K_{n}\right)<\Phi_{m, n}\left(\hat{K}_{n}\right)$ for some $m, n$.

Now, we can state the following.

Theorem 1 If every $\boldsymbol{\Omega}_{n}(\boldsymbol{P})$ is strictly monotonically increasing, then every $\boldsymbol{K} \in \Gamma$ is a Pareto optimal solution.

Proof: We separate our proof into the two different solution types: 1) optimal solutions and 2) trade-off solutions. In case 1$), F_{m, n}=0, \forall m, n$. Since all $\Phi_{m, n}$ are convex, no 
solution can lead to some $m, n$ with a smaller $\Phi_{m, n}$. For tradeoff solutions 2), we have at least two counteracting forces or rather counteracting KPIs for at least one parameter $m$. Thus, we have at least one $m, n$ for which $\frac{\partial \Phi_{m, n}}{\partial K_{n}}>0$ and at least one $m^{\prime}, n^{\prime}$ for which $\frac{\partial \Phi_{m^{\prime}, n^{\prime}}}{\partial K_{n^{\prime}}}<0$. We can only decrease the potential at the counteracting KPIs, since all other KPIs are within their desired value range leading to the fact, that their potential cannot be decreased. In order to decrease a particular counteracting KPI, we need to adjust some parameters. Since we define that all $\boldsymbol{\Omega}_{n}(P)$ are strictly monotonically increasing we have to decrease a parameter, if $\frac{\partial \Phi_{m, n}}{\partial K_{n}}>0$ (and vice versa) in order to decrease the corresponding potential. However, by applying such a parameter change we always increase the potential of the counteracting $\mathrm{KPI}(\mathrm{s})$ because the derivative of their potential has the opposite sign. Thus, all trade-off solutions must be Pareto optimal solutions.

Remark 1 Please note that we have modified the definition of a Pareto optimal solution in [8] slightly, in order for it to be applicable to the problem at hand.

Remark 2 If we consider $\boldsymbol{\Omega}_{n}(\boldsymbol{P})$ to be monotonically increasing instead of strictly monotonically increasing not all $\boldsymbol{K} \in \Gamma$ must be Pareto optimal solutions. Let us consider the example of two counteracting KPIs, $K_{1}$ and $K_{2}$, which corresponding forces act on the same parameter $P$ and cancel each other out, which implies that $\boldsymbol{K} \in \Gamma$. If for some reason, $\frac{\partial \boldsymbol{\Omega}_{1}(P)}{\partial P}=0$ for some interval around $\mathrm{P}$, then we can decrease the potential of $K_{2}$ without increasing the potential of $K_{1}$. Nevertheless, a more advanced algorithm could also take such situations into account leading to a weaker condition in Theorem 1 .

\section{E. Discussion}

If there exist mappings $\boldsymbol{\Omega}_{n}$ that are not strictly monotonic increasing for a small parameter range $\mathcal{X}^{\prime} \subset \mathcal{X}$, then the convergence condition may be violated in this parameter range. Thus, the overall weighted potential may increase for the iterations in which $\boldsymbol{P}^{j} \in \mathcal{X}^{\prime}$. However, after the not strictly monotonic increasing parameter range is passed the overall weighted potential will resume its decreasing trend. Following this, we infer that algorithm $\mathcal{A}$ can converge to a $\boldsymbol{K} \in \Gamma$ even though the convergence condition in Eq. (7) is violated for some iterations $j$. Note, that a non-increasing parameter range $\mathcal{X}^{\prime}$ will be passed always because the corresponding force will act in $\mathcal{X}^{\prime}$ as well and will not be weakened because $\boldsymbol{\Omega}_{n}$ is not increasing. Investigating the influence of $\boldsymbol{\Omega}$ on the convergence behaviour of the concept proposed will be a subject of future research. As another subject of future work, we can extend the force definition (see Eq. (3)) by a factor $\boldsymbol{R}(\boldsymbol{\Omega}, \ldots)$. In $\boldsymbol{R}(\boldsymbol{\Omega}, \ldots)$ we can apply automatic control techniques which compensate a disadvantageous behaviour of $\Omega$, in order to ensure that the convergence condition in Eq. (7) is always satisfied. Furthermore, we can introduce an explicit interconnection between various cells. One way to realize such an interconnection is to consider the potentials to be a function of all KPIs, i.e. $\Phi_{m, n}=\Phi_{m, n}(\boldsymbol{K})$. In this way, we can define optimization goals at cell $l$ which depend on a KPI in cell $l^{\prime}$. Correspondingly, the definition of a force would be
$F_{m, n}:=-\gamma_{m, n} \sum_{i=1}^{N L} \nabla_{K_{i}} \Phi_{m, n}(\boldsymbol{K})$. One can verify, that for this case the convergence condition in Eq. (7) becomes weaker since another sum appears:

$$
\sum_{r=1}^{M L} \sum_{n=1}^{N} \sum_{m=1}^{M L}\left[\sum_{i=1}^{N L} \gamma_{m, n} \frac{\partial \boldsymbol{\Omega}_{i}}{\partial P_{r}} \frac{\partial \Phi_{m, n}}{\partial K_{i}} \cdot F_{r}\right]<0 .
$$

Furthermore, we want to point out that the proposed concept equals a gradient search, if $M=N=1$ at each cell. From the authors point of view, the concept's main advantage is that it follows a joint optimization approach while maintaining a relatively low complexity level. However, this advantage comes at the cost of a rather stringent convergence condition. Moreover, the concept can balance multiple counteracting KPIs efficiently and enables a seamless adjustment of the resulting trade-offs according to operator-dependent priorities.

\section{Joint Optimization of Tilts And Cell Selection POLICIES}

\section{A. System Model}

The larger part of the system model used here has been adopted from [3]. Thus, the system model assumptions are presented in an abbreviated form. We model the downlink of a wireless network consisting of $\frac{L}{3}$ three-fold sectorized base stations ( $L$ equals the number of cells) serving an area $\mathcal{R} \subseteq \mathbb{R}^{2}$. The user locations are assumed to be randomly distributed according to a distribution $\delta(u)$ with $\int_{\mathcal{R}} \delta(u) d u=1$. The network traffic is modelled at flow level, where the arrival of flow requests is represented by a Poisson process with intensity $\lambda$ in $\frac{1}{\mathrm{~s} \cdot \mathrm{km}^{2}}$. The flow size is exponentially distributed with a mean $\Psi$. We can write the traffic intensity as $\sigma(u)=\lambda \Psi \delta(u)$ in $\frac{\mathrm{Mbit}}{\mathrm{s} \cdot \mathrm{km}^{2}}$. The signal-to-interference-andnoise-ratio $\alpha(u, \boldsymbol{\eta})(\mathrm{SINR})$ is calculated as

$$
\alpha(u, \boldsymbol{\eta})=\left\{\begin{array}{cc}
\frac{P_{\mathrm{rx}, l}(u)}{\sum_{t \neq l} \eta_{t} P_{\mathrm{rx}, t}(u)+\theta} & P_{\mathrm{rx}, l} \geq P_{\mathrm{rx}, \min } \\
0 & \text { otherwise }
\end{array}\right.
$$

where $P_{\mathrm{rx}, l}(u)$ equals the power received from BS $l$ at location $u, P_{\mathrm{rx}, \min }$ equals the minimum signal power, $\eta_{t}$ denotes the load of BS $t, \boldsymbol{\eta}$ denotes the load vector $\boldsymbol{\eta}=\left(\eta_{1}, \ldots, \eta_{L}\right)^{T}$, and $\theta$ accounts the noise power. The achievable data rate $c_{l}(u, \boldsymbol{\eta})$ is modelled according to the Shannon capacity. We assume the users to have the same location over the duration of the flow. Furthermore, we assume a round robin scheduler. In our model, the cell selection is simplified in comparison to [3], i.e. a user connects to BS $l$ if $l=s(u):=\operatorname{argmax}_{t} P_{\mathrm{rx}, t}(u)$. The corresponding cell areas are independent of $\boldsymbol{\eta}$ and can be written as $\mathcal{L}_{l}:=\left\{u \mid l=s(u), P_{\mathrm{rx}, l} \geq P_{\mathrm{rx}, \min }\right\}$. The load of cell $l$ is modelled as

$$
\eta_{l}=f_{l}(\boldsymbol{\eta}):=\int_{\mathcal{L}_{l}} \frac{\sigma(u)}{c_{l}(u, \boldsymbol{\eta})} d u .
$$

Eq. (12) only gives an implicit formulation of $\eta_{l}$. Thus, we obtain $\boldsymbol{\eta}$ via the fixed point iteration $\boldsymbol{\eta}^{k+1}:=\boldsymbol{g}\left(\boldsymbol{\eta}^{k}\right), k=$ $1,2, \ldots$ with $\boldsymbol{g}\left(\boldsymbol{\eta}^{k+1}\right):=b^{k} f\left(\boldsymbol{\eta}^{k}\right)+\left(1-b^{k}\right) \boldsymbol{\eta}^{k}$ and some $b^{k} \in[0,1]$. The convergence of this iteration is proven in [3]. We calculate the overall signal attenuation as $\chi=\chi_{\mathrm{PL}}+\chi_{\mathrm{Beam}}$, 


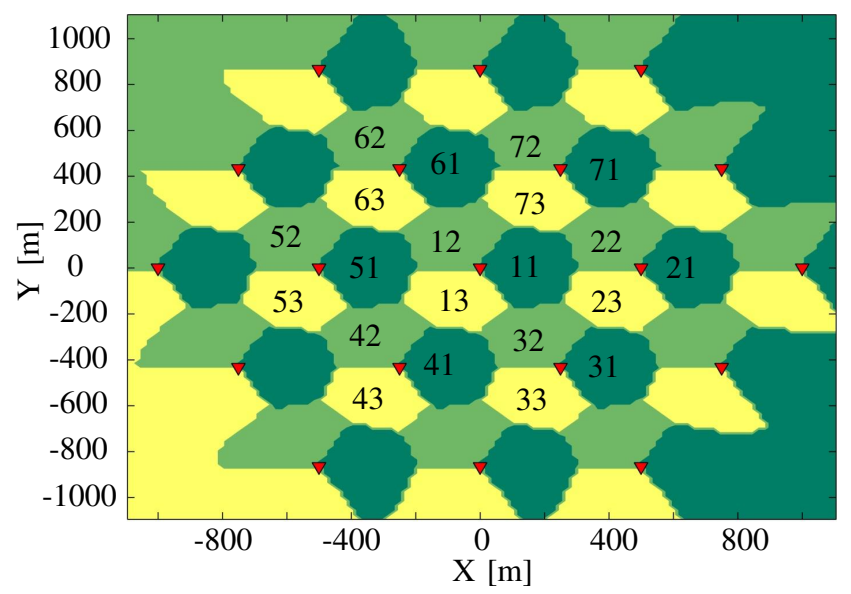

Fig. 2. Considered scenario layout consisting of 19 sites, each of them with 3 sectors. The various colours account the different cells, the red triangles depict the sites. All cells considered in the simulation are numbered.

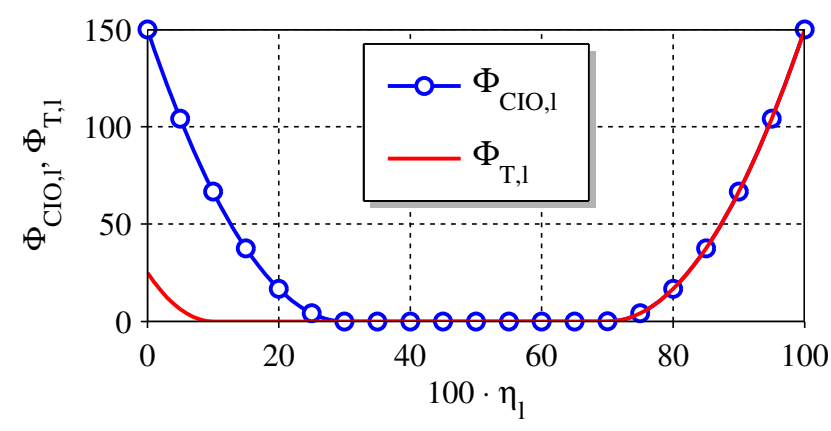

Fig. 3. The potentials used in the simulation. This potentials have been used at every cell $l$, the units are arbitrary.

where the path loss $\chi_{\mathrm{PL}}$ is calculated using the 3GPP path loss model for the urban scenario and macro sites [9]. We model the 3D beam pattern as a superposition of a $2 \mathrm{D}$ horizontal and 2D vertical pattern [10]: $\chi_{\text {Beam }}=B_{\text {azi }}+B_{\text {ele }}$. Both elevation and azimuth pattern are taken from [11].

\section{B. Simulation Scenario}

In the simulation presented in this paper, we employ the force field approach introduced above in order to balance a high load scenario by adjusting the cell individual offsets (CIOs) and the tilts $T$. Fig. 2 shows the scenario layout. We assume a regular hexagonal grid with 19 sites. Specifications for the network considered are presented in TABLE I. For the simulation, we employ algorithm $\mathcal{A}$ as presented above. The potentials are chosen such that the optimization goal is to avoid highly loaded cells $(\eta>0.7)$ and to prevent cell loads under $30 \%$. All potentials are with respect to the load. Thus, we change the assignment of the indices as follows: The first index defines the parameter-type and the second index assigns the cell. For example, $\Phi_{\mathrm{T}, 11}$ denotes the potential with respect to the tilt at cell 11 . We generate a high load scenario at cells 11,12 , and 13 , by setting the arrival intensity at these cells to $\lambda_{\text {high. }}$ At all other cells the arrival intensity is $\lambda_{\text {low }}$.
TABLE I

CONSIDERED SIMULATION PARAMETERS

\begin{tabular}{lr}
\hline System Parameters & \\
\hline \hline Carrier Frequency & $2.0 \mathrm{GHz}$ \\
Bandwidth & $10 \mathrm{MHz}$ \\
\hline BS Transmit Power & $46 \mathrm{dBm}$ \\
BS Height & $15 \mathrm{~m}$ \\
Backward Attenuation $B_{0}$ & $20 \mathrm{dBm}$ \\
BS Antenna Gain & $14 \mathrm{dBi}$ \\
Thermal Noise $\theta$ & $-104 \mathrm{dBm}$ \\
Terminal Receiver Sensitivity $P_{\mathrm{rx}, \mathrm{min}}$ & $-100 \mathrm{dBm}$ \\
Terminal Height & $1.5 \mathrm{~m}$ \\
\hline Initial Tilt $T_{l}$ & $7^{\circ} \forall l$ \\
Mean Flow Size $\Psi$ & $500 \mathrm{kbit}$ \\
Arrival Intensity $\lambda_{\text {low }}$ & $5200 \mathrm{~s}^{-1} \cdot \mathrm{km}^{-2}$ \\
Arrival Intensity $\lambda_{\text {high }}$ & $16500 \mathrm{~s}^{-1} \cdot \mathrm{km}^{-2}$ \\
\hline Spatial Resolution & $20 \mathrm{~m}$ \\
\hline
\end{tabular}

\section{Simulation Results}

For the evaluation of the simulation, we consider the sets $\eta_{\text {high }}=\left\{\eta_{11}, \eta_{12}, \eta_{13}\right\}$ and $\eta_{\text {low }}=\left\{\eta_{21}, \eta_{22}, \eta_{23}, \eta_{31}, \ldots \eta_{73}\right\}$, where $\eta_{a b}$ denotes the load at cell $a b$. Moreover, we evaluate the 5th percentile of the $\operatorname{SINR~}_{5}(\alpha)$ and the overall weighted potential $\varphi$ of the cells $11,12, \ldots, 73$. In all simulations, we set $\gamma_{\mathrm{T}, l}=\frac{1}{25}, \forall l$ while we diversify $\gamma_{\mathrm{CIO}, l}=\left\{0, \frac{1}{40}, \frac{1}{25}\right\}, \forall l$. In Fig. 4, we present the progress of the maximum cell load among the high load area, i.e. $\max \left(\eta_{\text {high }}\right)$ and the maximum cell load among the surrounding cells, i.e. $\max \left(\eta_{\text {low }}\right)$. It is clearly visible that the algorithm shifts the traffic from a highly loaded area to the neighbouring cells. We can see that the load balancing becomes faster by increasing usage of the CIOforce (increasing $\gamma_{\mathrm{CIO}, l}$ ). Nevertheless, the system converges to the same situation for both versions, $\gamma_{\mathrm{CIO}, l}=0.04$ and $\gamma_{\mathrm{CIO}, l}=0.025$. If the CIOs are not modified $\left(\gamma_{\mathrm{CIO}, l}=0\right.$ leads to $F_{\mathrm{CIO}, l}=0$ ), it is not possible to balance the high load situation. For $\gamma_{\mathrm{CIO}, l}=\{0.025,0.04\}$, the system reaches an almost completely balanced situation after 43 iterations. In this situation, cell 11 with a load of 0.73 is confronted with loads of $0.73,0.66,0.72$, and 0.67 at the cells $22,23,32$, and 73 . But why are some loads not below $70 \%$ ? Let us postpone this discussion for a minute, in order to analyse the behaviour of the 5 th percentile of the $\operatorname{SINR~}_{5}(\alpha)$ and the overall weighted potential $\varphi$. The progress of the 5th percentile of the SINR $\mathrm{Q}_{5}(\alpha)$ is shown in Fig. 5. We can see that the 5th percentile becomes worse in all cases. If we observe only the first 15 iterations, we can see that the 5 th percentile decreases as the adjustment of the CIOs increases, i.e. as $\gamma_{\mathrm{CIO}, l}$ increases. To us, this behaviour seems reasonable since adjusting the CIOs pushes cell edge users into cells where they experience a lower SINR. However, starting from iteration 15 the scenarios $\gamma_{\mathrm{CIO}, l}=\{0.025,0.04\}$ outperform the case where $\gamma_{\mathrm{CIO}, l}=0$. Obviously, the SINR can also be considerably worsened by adjusting the tilt. Thus, we can infer from Fig. 5, that a well-matched trade-off between the usage of tilts and CIOs is required in order to harm the 5 th percentile of the SINR $\mathrm{Q}_{5}(\alpha)$ as little as possible. In Fig. 6, we present the progress of 


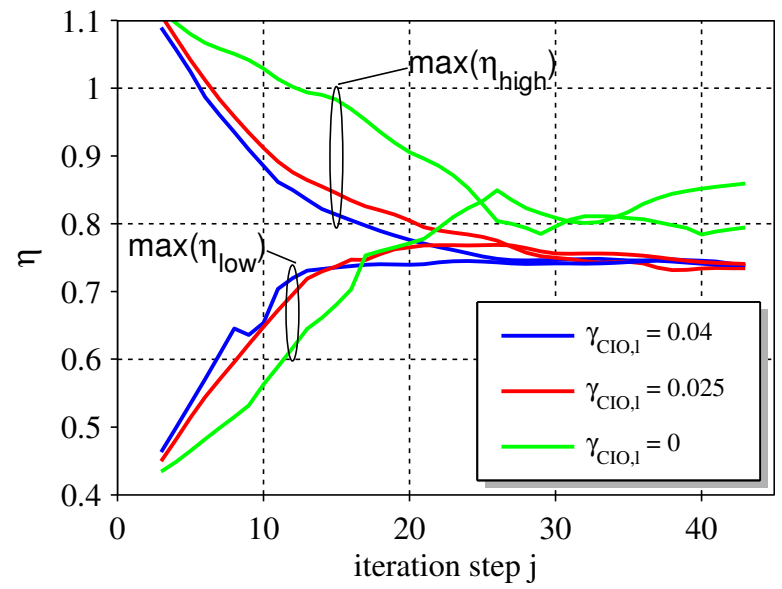

Fig. 4. The progress of $\max \left(\eta_{\text {high }}\right)$ and $\max \left(\eta_{\text {low }}\right)$ during the simulation.

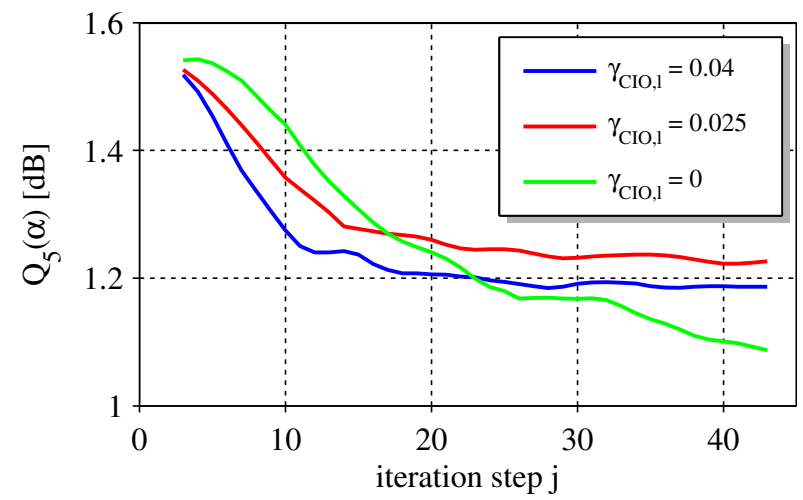

Fig. 5. The progress of the 5 th percentile of the $\operatorname{SINR} \mathrm{Q}_{5}(\alpha)$ during the simulation.

the overall weighted potential $\varphi$ during the simulation. Again, we can conclude that adjusting just the tilts is not favourable. Even though the overall weighted potential $\varphi$ is decreased considerably in the scenarios $\gamma_{\mathrm{CIO}, l}=\{0.025,0.04\}$, it does not converge to zero. As already mentioned above, some cells still have loads approx. $2-3 \%$ higher than $70 \%$. This causes the residual potential. From the authors point of view, this residual overload is caused by 1 ) the spatial quantization with a resolution of $20 \times 20 \mathrm{~m}, 2$ ) a high load scenario that is rather difficult to handle, and 3 ) the small scaling factors. The spatial quantization leads to the fact, that only $20 \times 20 \mathrm{~m}$ planes can change the sector from which they are served. Hence, it is not possible to adjust the loads precisely. However, this is only a computational issue. Due to a difficult high load scenario $\left(\max \left(\eta_{\text {high }}^{j=0}\right)=1.19\right)$ we also reach the upper load limit $(70 \%)$ at cells next to the high load hot spot after balancing. From point 3 ) it follows that the system converges rather slowly.

\section{CONCLUSION}

In this work, we introduced a novel force field based joint optimization concept. Basis of the concept are potentials which

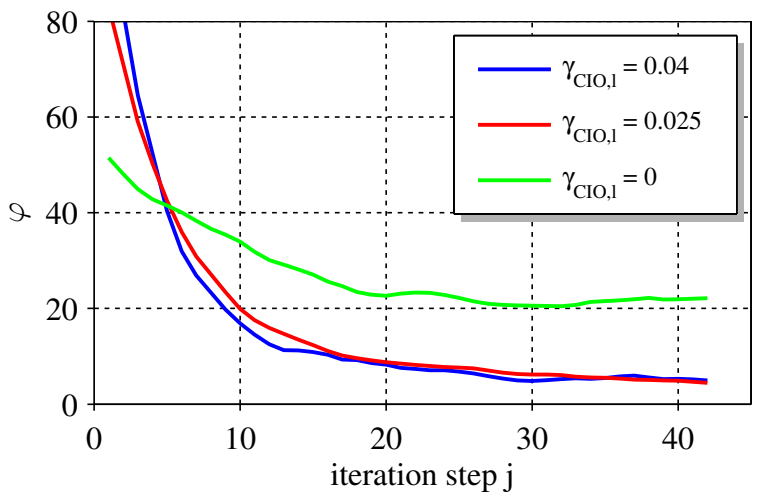

Fig. 6. The progress of the overall weighted potential $\varphi$ during the simulation. The units are arbitrary.

define the optimization goals and induce acting forces. These forces modify the network's parameters in order to balance all KPIs considered. Forces which act on one and the same parameter interfere with each other. Resulting trade-offs between counteracting KPIs are Pareto optimal under given conditions and can be regulated seamlessly by means of scaling factors. Moreover, we created an algorithm based on the force field approach. We also defined a solution set and obtained a general convergence condition. The algorithm always converges to a point in the solution set if the convergence condition is satisfied. We employed the algorithm created for balancing a high load scenario by concurrently adjusting the tilts and the CIOs.

\section{REFERENCES}

[1] L. C. Schmelz, van den Berg et al. (2008) Requirements for selforganising networks. [Online]. Available: http://www.fp7-socrates.org

[2] - (2008) Framework for the development of self-organisation methods. [Online]. Available: http://www.fp7-socrates.org

[3] H. Klessig, A. Fehske et al., "Improving Coverage and Load Conditions Through Joint Adaptation of Antenna Tilts and Cell Selection Rules in Mobile Networks," International Workshop on Self-Organizing Networks at the International Symposium on Wireless Communication Systems, Paris, France, 2012.

[4] F. Richter and G. Fettweis, "Base Station Placement Based on Force Fields," IEEE 75th Vehicular Technology Conference: VTC2012-Spring, 2012.

[5] D. G. Luenberger, "Zangwills Global Convergence Theorem," Linear and Onlinear Programming, vol. 2, 2003.

[6] J. Heinonen, Lecutres on Analysis on Metric Spaces. Springer, 2001.

[7] D. P. Bertsekas, Nonlinear Programming. Belmont, MA: Athena Scientific, 1999.

[8] M. Ehrgott, Multicriteria Optimization. New York: Springer-Verlag, 2005.

[9] Technical Specification Group Radio Access Network, "Further advancements for e-utra: Physical layer aspects (release 9)," 3rd Generation Partnership Project, Tech. Rep. TR 36.814, 2009.

[10] R. Hoppe, "Comparison and evaluation of algorithms for the interpolation of $3 \mathrm{~d}$ antenna patterns based on $2 \mathrm{~d}$ horizontal and $2 \mathrm{~d}$ vertical patterns," 2003.

[11] Technical Specification Group Radio Access Network, "Physical layer aspects for evolved universal terrestrial radio access (utra)," 3rd Generation Partnership Project, Tech. Rep. TR 25.814, 2006. 